\title{
Ultra-high energy cosmic rays and the strongest AGN flares
}

\author{
Maxim Pshirkov ${ }^{1,2,3, *}$ and Bulat Nizamov ${ }^{1,}$ \\ ${ }^{1}$ Sternberg Astronomical Institute, Lomonosov Moscow State University, Universitetsky prospekt 13, \\ 119992, Moscow, Russia \\ ${ }^{2}$ Institute for Nuclear Research of the Russian Academy of Sciences, 60th October Anniversary st. 7a, \\ 117312, Moscow, Russia \\ ${ }^{3}$ P.N. Lebedev Physical Institute, Pushchino Radio Astronomy Observatory, 142290 Pushchino, Russia
}

\begin{abstract}
We perform an observational test of the hypothesis that ultra-high energy cosmic rays $\left(E>10^{20} \mathrm{eV}\right)$ are accelerated in giant flares of active galactic nuclei (AGN). We use the observations of Fermi LAT during the first 7.4 years of the mission. We select highly variable AGNs with the redshift $z \leq 0.3$ and use an empirical relation between the bolometric luminosity and the jet kinetic power in order to find the local kinetic emissivity of strong AGN flares: $\mathcal{L} \sim 1.1 \times 10^{45} \mathrm{erg} \mathrm{Mpc}^{-3} \mathrm{yr}^{-1}$. This is approximately one order of magnitude larger than the UHECR emissivity estimated from the data of Pierre Auger and Telescope Array observatories which makes the whole scenarion feasible provided the acceleration spectrum is sufficiently hard and/or narrow.
\end{abstract}

\section{Introduction}

The origin of the highest, or ultra-high energy cosmic rays (UHECR) remains an unsolved problem of modern astrophysics. On the one hand, the data of Pierre Auger (PAO) and Telescope Array (TA) observatories clearly show that there exist cosmic rays (CR) with energies in excess of $10^{20} \mathrm{eV}$. On the other hand, we do not know any sources within $\sim 100 \mathrm{Mpc}$ which could accelerate particles to such energies. Still, these sources should be local because of the strong reduction of CR propagation length above $6 \times 10^{19} \mathrm{eV}$ due to the interaction with CMB. This is known as Greisen-Zatsepin-Kuzmin effect $[1,2]$ which was discovered observationally by HiRes [3] and lately confirmed with a very high statistical significance by PAO and TA $[4,5]$. At the same time, the potential accelerator should posses rather extreme properties [6], namely a combination of the size $R$ and the magnetic field $B$ which satisfies the condition

$$
R B \gtrsim 3 \times 10^{17} \Gamma^{-1} E_{20}
$$

where $E_{20}$ is the CR energy in units $10^{20} \mathrm{eV}$. In the case of relativistic jets this translates to

$$
L \sim \frac{1}{6} c \Gamma^{4} B^{2} R^{2} \gtrsim 10^{45} \Gamma^{2} E_{20}^{2} \mathrm{erg} / \mathrm{s} .
$$

Summing up, there are two effects interplaying in the highest-energy part of the CR spectrum: progressively smaller active volume $V_{\mathrm{GZK}}$ and increasing degree of 'extremeness' of

*e-mail: pshirkov@sai.msu.ru 
the sources. As one does not know any appropriate steady sources within $V_{\mathrm{GZK}}$, the most natural way out is high energy transients. A candidate UHECR accelerator should satisfy two conditions. First, the Hillas criterion should be fulfilled and, second, the total energy output should be sufficiently large in order to explain the observed UHECR intensity. The total emissivity required by TA and PAO observations is $8 \times 10^{43}$ and $1.5 \times 10^{43} \mathrm{erg} \mathrm{s}^{-1} \mathrm{Mpc}^{-3}$ respectively.

The two promising candidates are gamma-ray bursts (GRB) and giant flares of active galactic nuclei (AGN) [7-9]. However, GRBs, while easily meeting the first criterion, apparently do not provide sufficient energy output which is $\sim 5 \times 10^{43} \mathrm{erg} \mathrm{s}^{-1} \mathrm{Mpc}^{-3}$ [10].

On the other hand, AGN flares with luminosities higher than $10^{50} \mathrm{erg} / \mathrm{s}$ have been observed, so that the question is whether such events occur sufficiently frequently in the local volume. To check this point, we have used $\gamma$-ray observations of Fermi LAT in the high energy (HE, $E>100 \mathrm{MeV}$ ) range. We have focused on the 'local sources' $(z<0.3)$ because we wanted to avoid complications that arise due to a redshift evolution but still retain a decent statistics, with a probed volume that is more than 1000 times larger than the GZK volume.

In this work we focus on the AGN flares as possible UHECR accelerators and perform an observational test of this possibility.

\section{Data and method}

Strong flares of AGNs can possibly accelerate protons to the very highest energies $[8,9]$. Stringent requirements on the size of the acceleration region and strength of the magnetic field there naturally translate into the lower limit on the 'magnetic luminosity' $L_{B}$ (e.g. [9]):

$$
L_{B} \geq 10^{45} \Gamma^{2} E_{20}^{2} \operatorname{erg~s}^{-1},
$$

which is adopted in this paper as a threshold value; $E_{20}$ is the UHECR energy in units of $10^{20} \mathrm{eV}$. Such extreme flares have never been observed in $V_{\mathrm{GZK}}$ (see below for the method of $L_{\mathrm{B}}$ estimation). Nevertheless it is possible to calculate their local energetics using much larger test volume $V_{0}$ : we have selected a sphere with a radius corresponding to $z=0.3, V_{0}>$ $10^{3} V_{\text {GZK }}$. This volume is large enough to avoid significant statistical fluctuations and still it is appropriate to neglect effects of cosmological evolution at $z<0.3$. Firstly, we selected all flaring sources satisfying Eq. (3), that potentially could be the sites of UHECR acceleration. It is impossible to directly measure $L_{B}$, so we used bolometric luminosity $L_{\text {bol }}$ as a proxy. That implies that energy density of the magnetic field and electrons are close, $\epsilon_{\mathrm{B}} \sim \epsilon_{\mathrm{e}}$ and AGN jets radiate efficiently.

The whole approach is made feasible by the fact that the bolometric luminosity can be rather reliably estimated using only the high-energy luminosity $L_{\gamma}, 0.1 \mathrm{GeV}<E_{\gamma}<$ $100 \mathrm{GeV}[11]$ :

$$
L_{\text {bol }} \sim 2 L_{\gamma} .
$$

The spectral-energy distribution of the blazars consists of two peaks. The first one lies around optical frequency range, and the other one is usually in the HE region; the luminosities in two peaks are comparable. This justifies the adoption of the factor 2 in the Eq. (4). There can be a slight overestimation of this coefficient due to the fact that for the sources being in the high state the second peak becomes more pronounced.

For almost 10 years Fermi LAT has been continuously observing the celestial sphere at energies $>100 \mathrm{MeV}$. That allowed us to compile the full census of the very bright flares in the local Universe. We have made use of the second catalog of flaring gamma-ray sources (2FAV) based on Fermi All-Sky Variability Analysis (FAVA) [12]. This catalog is a collection of gamma-ray sources which show significantly higher (or lower) photon flux in a given 
time window as compared to what could be expected from the average flux from the source direction. The catalog is based on the Fermi observations during the first 7.4 years of the mission.

Among the AGNs in 2FAV we selected those within the test volume $V_{0}$. Moreover, it is possible that some AGNs could be in high state for a time span longer than 7.4 years and therefore enter the catalog as steady sources. In order not to miss such objects, we checked 3FGL catalog for superluminous sources within $z=0.3$. For the sources with known redshift we approximated the isotropic equivalent bolometric luminosity with (4) where $L_{\gamma} \sim$ $4 \pi d^{2} F_{100}, d$ is the distance to the source and $F_{100}$ is the energy flux above $100 \mathrm{MeV}$ taken from 3FGL. Only one source with the known $z \leq 0.3$, S5 0716+71, exceeded the threshold (3). This source is also in 2FAV catalog and we included it in our analysis. The final list of selected AGNs consists of thirty nine sources.

The most rigorous way to calculate the gamma-ray flux is to use maximum likelihood method (ML) implemented in the Fermi Science Tools utility gtlike. However, the total time span of the intervals to be analyzed is rather large which makes this approach difficult. Instead, we used aperture photometry (AP) light curves provided by SSDC website ${ }^{1}$. This simple approach does not distinguish between source and background photons, therefore the flux is inevitably overestimated, especially at low galactic latitudes. Also, at the energies $\sim 100 \mathrm{MeV}$ the point spread function of LAT is wide so that many sources contribute to the measured flux. To avoid these problems, we used AP light curves in the range 1-100 GeV within $2^{\circ}$ from each source and extrapolated them down to $0.1 \mathrm{GeV}$ in the assumption of a flat spectrum. We check the correctness of such a procedure in two steps. First, we compare the fluxes in the range $1-100 \mathrm{GeV}$ obtained by AP and ML for a number of time intervals. It appears that AP is mostly consistent with ML within the statistical uncertainties Second, we estimate what error can be introduced to our estimations by the flux extrapolation to the range $0.1-100 \mathrm{GeV}$ in the assumption of a flat source spectrum and find that it underestimates the flux by up to $40 \%$ for typical blazar spectra.

The threshold luminosity given by Eq. (3) depends on the bulk Lorentz factor of the jet. We used Lorentz factor $\Gamma=10$ as a robust benchmark value [13-15] and considered acceleration to energies higher than $10^{20} \mathrm{eV}$. These parameters set the threshold (3) at the level of $10^{47} \mathrm{erg} / \mathrm{s}$. We have explicitly checked that the acceleration in this regime was not limited by the synchrotron losses. Due to a relatively long duration of flares $\left(>10^{5} \mathrm{~s}\right)$ constraints on the minimal size of the acceleration region are greatly relaxed, which in turn allows to decrease magnitude of needed magnetic field and, therefore, importance of synchrotron losses, see e.g. eqs. (1) and (2) in [9]. Next, we selected the time intervals where the estimated luminosity exceeds the threshold, i.e. a given AGN can accelerate UHECRs. As a proxy of the bolometric luminosity we use the radiative energy flux in the range $0.1-100 \mathrm{GeV}$ multiplied by a factor of 2 (see (4)).

Now it is possible to estimate total kinetic energy of these flares: within the chosen intervals we convert gamma-ray luminosity to the jet kinetic power as proposed by [11]:

$$
\log P_{\text {jet }}=A \log L_{\gamma}+B
$$

We assume that, due to the beaming of the photons, we can not observe all blazars, but only a fraction of order $\Omega_{b} / 2 \pi \sim 1 / 2 \Gamma^{2}$ where $\Omega_{b}$ is the surface angle of each of the two jets. Therefore in order to obtain the estimate of the total kinetic energy within the test volume we multiply $P_{\text {jet }}$ by $2 \Gamma^{2}$. The kinetic energy thus obtained is divided by the time of observation (7.4 years) and by the volume $V_{0}$ which gives the total kinetic emissivity. In Tab. 1, we show the sources which give non-zero contributions to the total kinetic emissivity.

\footnotetext{
${ }^{1}$ http://www.asdc.asi.it/
} 
Table 1. The list of the analyzed AGNs. $z, l, b$ are the redshift and galactic longitude and latitude, $N_{\text {flare }}$ is the number of flares, $E_{\text {kin }}$ is the contribution of the source to the total kinetic emissivity, $L_{\max }$ is the maximum isotropic equivalent bolometric luminocity in the observation period, in units of $10^{47} \mathrm{erg} \mathrm{s}^{-1}$.

\begin{tabular}{lcccccc} 
Name & $z$ & $l$ & $b$ & $N_{\text {flare }}$ & $E_{\text {kin }}$ & $L_{\text {max }}$ \\
\hline ON 246 & 0.14 & 232.8 & 84.9 & 2 & $1.1 \times 10^{40}$ & 1.20 \\
3C 273 & 0.16 & 290.0 & 64.4 & 3 & $3.5 \times 10^{40}$ & 2.78 \\
PKS 0736+01 & 0.19 & 217.0 & 11.4 & 5 & $5.8 \times 10^{40}$ & 2.71 \\
MG1 J021114+1051 & 0.20 & 152.6 & -47.4 & 2 & $1.1 \times 10^{40}$ & 1.43 \\
OX 169 & 0.21 & 72.1 & -26.1 & 1 & $5.3 \times 10^{39}$ & 1.13 \\
1H 1013+498 & 0.21 & 165.5 & 52.7 & 2 & $1.9 \times 10^{40}$ & 2.00 \\
B2 2023+33 & 0.22 & 73.1 & -2.4 & 36 & $2.1 \times 10^{42}$ & 3.22 \\
PMN J0017-0512 & 0.23 & 101.2 & -66.6 & 1 & $5.8 \times 10^{39}$ & 1.39 \\
PMN J1903-6749 & 0.26 & 327.7 & -26.1 & 1 & $6.3 \times 10^{39}$ & 1.62 \\
PKS 0301-243 & 0.26 & 214.6 & -60.2 & 10 & $8.2 \times 10^{40}$ & 7.42 \\
S2 0109+22 & 0.27 & 129.1 & -39.9 & 31 & $3.1 \times 10^{41}$ & 2.92 \\
PMN J1802-3940 & 0.30 & 352.5 & -8.4 & 61 & $9.6 \times 10^{41}$ & 5.06 \\
NVSS J223708-392137 & 0.30 & 0.59 & -59.6 & 2 & $1.2 \times 10^{40}$ & 1.55 \\
S5 0716+71 & 0.30 & 144.0 & 28.0 & 42 & $2.1 \times 10^{42}$ & 8.73
\end{tabular}

In this table $N_{\text {flare }}$ is the number of flares detected in a given source ${ }^{2}$. The total emissivity

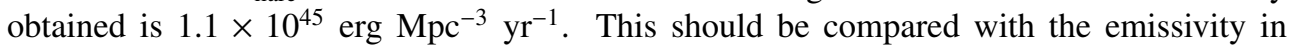
UHECRs. It was shown that the emissivity required to reproduce the UHECR data above $10^{18}$ $\mathrm{eV}$ should be of the order $10^{45}-10^{46} \mathrm{erg}^{\mathrm{Mpc}^{-3}} \mathrm{yr}^{-1}$ [16] which corresponds to $\sim 10^{43}-10^{44}$ erg $\mathrm{Mpc}^{-3} \mathrm{yr}^{-1}$ above $10^{20} \mathrm{eV}$. Our own calculation which follows the approach of [17] using the photo-pion production cross-section from [18] gave the values of $7.5 \times 10^{43} \mathrm{erg} \mathrm{Mpc}^{-3}$

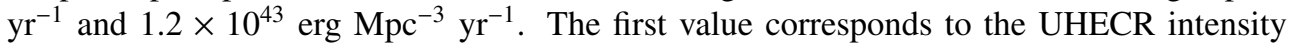
as observed by Telescope Array collaboration [5], and the second value corresponds to the intensity reported by the Pierre Auger collaboration [19]. Thus, the ratio of the UHECR emissivity to the AGNs' jet kinetic power, according to our estimations, varies from $1.1 \times 10^{-2}$ in case of the lower UHECR flux of Pierre Auger to $6.8 \times 10^{-2}$ in case of the higher UHECR flux of Telescope Array. The threshold (3) is not a well-defined quantity. We estimated the total kinetic luminosity as described in Section 2 with the threshold luminosities of $3 \times 10^{46}$ $\mathrm{erg} / \mathrm{s}$ and $3 \times 10^{47} \mathrm{erg} / \mathrm{s}$. The values obtained are $2.4 \times 10^{45} \mathrm{erg} \mathrm{Mpc}^{-3} \mathrm{yr}^{-1}$ and $1.8 \times 10^{44} \mathrm{erg}$ $\mathrm{Mpc}^{-3} \mathrm{yr}^{-1}$. The latter value is very close to the inferred UHECR emissivity which can make this scenario problematic.

\section{Discussion}

In the previous section we have calculated emissivity in UHECRs $\mathcal{L}_{\text {UHECR }}=7.5 \times$ $10^{43} \mathrm{erg} \mathrm{Mpc}^{-3} \mathrm{yr}^{-1}$ or $1.2 \times 10^{43} \mathrm{erg} \mathrm{Mpc}^{-3} \mathrm{yr}^{-1}$ from the observed flux (reported by Telescope Array or Pierre Auger) of these particles and compared it with a kinetic emissivity in

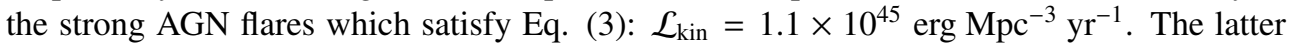
one was calculated taking into account the fact that we can observe only small fraction of all flaring sources and total observed kinetic emissivity should be multiplied by the number of unseen sources, $n=2 \pi / \Omega_{b} \sim 2 \Gamma^{2}$. The viability of the scenario crucially depends on the degree of UHECR beaming, $\Omega_{\mathrm{UHECR}}$. If the accelerated UHECRs remain strongly beamed

\footnotetext{
${ }^{2}$ We call a 'flare' each time interval when a source exceeds the threshold (3).
} 
Figure 1. Constraints on the spectrum of the UHECRs produced in extreme AGN flares. The spectrum is described by a power law: $d N / d E \sim E^{\alpha}, E_{\min }(\alpha)<E<E_{\max }=10^{21} \mathrm{eV}$. The upper/lower curve corresponds to the TA/PAO energy spectrum parametrization.

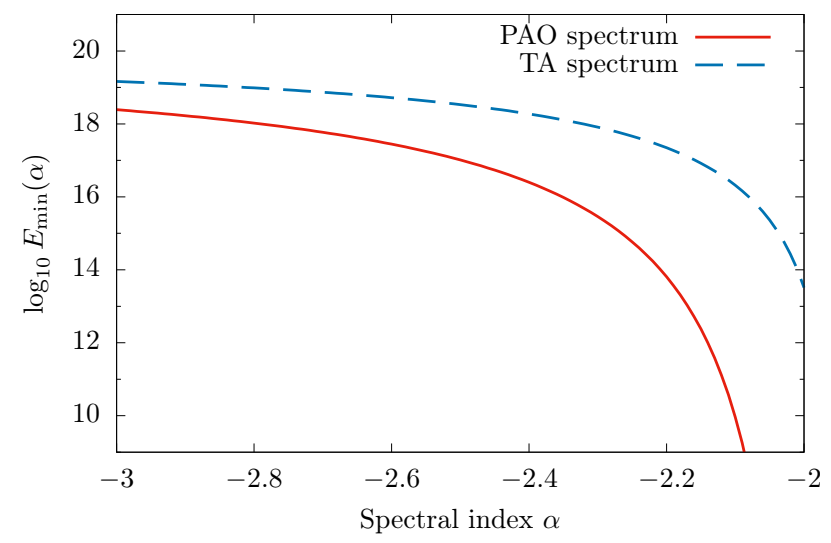

with $\Omega_{\text {UHECR }} \sim \Omega_{b}$, the UHECR emissivity $\mathcal{L}_{\text {UHECR }}$ will be corrected for the beaming factor as well. As after this correction $\mathcal{L}_{\text {UHECR }}$ will be much larger than $\mathcal{L}_{\text {kin }}$ it would clearly make the model unfeasible.

The UHECRs can be effectively isotropised during their propagation towards the observer, increasing $\Omega_{\text {UHECR }}$ up to value of order unity. The isotropisation can take place either in the immediate vicinity of the flaring region, which in turn shall be very close to the AGN engine, or in the magnetized intracluster medium. Large values of $\Omega_{\text {UHECR }}$ mean that the observed flux of cosmic rays is generated by large number of flares with only small fraction of them pointing at us.

Even then, the luminosity in the UHECRs with $E>10^{20} \mathrm{eV}$ amounts to a sizable fraction of the full kinetic luminosity of suprathreshold AGN flares in the local Universe, $1.1 \times 10^{-2}$ or $6.8 \times 10^{-2}$ depending on the UHECR spectrum selected, either PA or TA. Nevertheless, from the point of view of pure energetics it is not impossible that these flares can be the primary sources of the CRs of the highest energies. More than that, relatively large value of the ratio of UHECR to the full kinetic luminosity allows to put stringent constraints on the properties of the spectrum of CRs produced in the flares. We demanded that the total amount of energy in these CRs will not exceed one half of the kinetic energy [20]. The spectrum of the UHECRs was described as a simple power-law, $\propto E^{-\alpha}, E_{\min }<E<E_{\max }=$ $10^{21} \mathrm{eV}$ bounded from above and below. The results in form of $E_{\min }(\alpha)$ curves are presented in the Fig. 1. We have also checked that the results are not considerably changed with an increased value of $E_{\max }=10^{22} \mathrm{eV}$. It can be seen that soft extended spectra are excluded with a high degree of confidence, and the spectrum of produced UHECR must be sufficiently hard and/or narrow. A lot of models predict the very same shape for the spectrum of particles escaping from relativistic collisionless shocks (e.g., [21-23]) or accelerated immediately in BH magnetospheres [24].

Additional constraints on the spectral shape can be obtained using multiwavelength and multimessenger observations - large regions of parameter space can be excluded depending on realized scenario of the cosmic source evolution [25]. This question with detailed treatment of the redshift dependence of AGN flares energetics will be studied elsewhere.

Small number of observed events with $E>10^{20} \mathrm{eV}$ does not allow to perform meaningful large-scale anisotropy studies, it is still impossible to reliably distinguish between isotropic or 
some anisotropic distributions. Nevertheless, it is safe to claim that at any given moment the number of active sources in the $V_{\mathrm{GZK}}$ must be larger or equal to one [26]. We have observed $N=14$ potential sources in $t=7.4$ years inside $V_{0}=10^{3} V_{\mathrm{GZK}}$ volume which gives the following estimate for the rate of extreme UHECR producing flares in the GZK volume:

$$
\mathcal{R} \sim \frac{N}{t} \times \frac{V_{\mathrm{GZK}}}{V_{0}} \Gamma^{2} \sim 0.2 \mathrm{yr}^{-1}
$$

Number of active UHECR sources can be readily estimated as

$$
\mathcal{N}=\mathcal{R} t_{\mathrm{UHECR}},
$$

where $t_{\mathrm{UHECR}}$ is the duration of observed UHECR signal. At first glance, given that the typical duration of gamma flares $t_{\gamma}$ are of order of weeks ${ }^{3}$, this low rate seems to be at variance with the observations. However, being charged particles, UHECRs are subject to considerable deflection in the magnetic fields; when propagating, short pulses are broadened by scattering:

$$
\tau \sim d \theta_{s}^{2} / 2 c,
$$

where $d$ is the propagation path length, $\theta_{s}$ is the characteristic angle of scattering. The observed duration $t_{\mathrm{UHECR}}=\tau$ can be much longer than $t_{\gamma}$. There are two closely related causes of scattering: first, there is a part due to the deflection in the random magnetic fields; second, there is considerable scattering due to a finite energy width of the pulse - cosmic rays at different energies are deflected by different angles.

There are several regions where scattering can possibly take place. At the very least, a pulse of UHECRs will be deflected in the Galactic magnetic fields. At these energies, the magnitude of scattering due to the random parts of the galactic magnetic field will be several tenth of degree for out of the Galactic plane directions [27], corresponding length is of order of kpc. Also the signal will be spread by several degrees in the regular galactic magnetic fields [28]. That translates to characteristic time scales $\tau$ around several decades. It could reach more than $10^{3} \mathrm{yr}$ if a dipole field is present [29]. Also the UHECRs will be scattered during inevitable isotropization - the corresponding timescales are highly uncertain, they could be very large if the UHECRs were scattered in the magnetic fields of clusters, or, on the other hand, they could be insignificantly small if the primary site of the isotropization was very close to the source. Another sites of possible scattering include the local filament of the Large scale structure [30] or the extragalactic voids [29, 31]. In the former case possible time delays could be of order $10^{5}$ years, when in the latter case they could exceed $10^{7}$ years. That means that at the highest energies we will simultaneously observe at least $O(10)$ sources and probably many more.

There are several underlying assumptions that are important to our approach and should be emphasized. First, we assume that the jets are radiatively efficient and magnetic field energy is in a rough equipartition with energy of relativistic electrons. Only that premise allows us to estimate magnetic luminosity from the observed bolometric luminosity, and in case of radiatively inefficient jet and/or large deviation of $\epsilon_{B} / \epsilon_{e}$ ratio from unity our estimate will be inaccurate. Still, our algorithm for search for extreme flares is likely to be on the conservative side, as one would expect that $\epsilon_{B} / \epsilon_{e}$ ratio rather deviates towards larger values, meaning that we underestimate the number of potential sources. Also some underestimation of real $\mathcal{L}_{\text {kin }}$ can be due to a number of blazars without assigned redshift. There are 65 associated sources

\footnotetext{
${ }^{3}$ Also one can not exclude the possibility that individual flares can emerge during much longer interval when the source is in a state of increased activity.
} 
without assigned redshift in the 2FAV catalog, 57 of them would satisfy Eq. 3 if they were situated at $z=0.3$. In the highly unlikely scenario that all sources without estimated redshifts reside so close inside $V_{0}$ volume it will shift upwards our estimate of $\mathcal{L}_{\text {kin }}$ by factor of 3 and, accordingly, the estimate of energy available for UHECR acceleration.

We have chosen the lightest, protonic, composition for the UHECRs as a limiting scenario. From the theoretical point of view, acceleration of protons to the highest energies is very difficult, i.e. if it is possible for some class of sources to accelerate them, then, a fortiori, these sources can potentially produce UHECR nuclei with larger atomic mass $A$. From the observational point of view, the composition of the UHECRs at the highest energies is still far from being certain: while the results of the Telescope Array experiment favor lighter one [32], the ones of Pierre Auger Observatory indicate that there is progressive increase in $A$ at higher energies [33], although the detailed analysis shows that there is a good agreement between distributions in shower depth maximum within the systematic uncertainties [34]. Still, even the PAO results are fully consistent with the existence of a large fraction of protons even at the energies $E>10^{19} \mathrm{eV}$. More than that, due to a very low statistics ${ }^{4}$ there is no information about UHECR composition at the energies $E>10^{20} \mathrm{eV}$, so it is not inconceivable that we will eventually observe decrease in $A$.

\section{Conclusions}

The full census of strong local gamma-ray flares at redshifts $z<0.3$ from the Fermi-LAT data was used in order to find ones that can possibly accelerate protons to the highest energies $E>10^{20} \mathrm{eV}$ and estimate maximal disposable amount of kinetic energy that can be potentially used for UHECR acceleration. The estimated kinetic emissivity is more than one order of magnitude higher than the UHECR emissivity obtained from the observations, which makes the scenario feasible from the point of view of total energetics, if the escape spectrum of cosmic rays is not too soft. Also, the number of potential sources in the Greisen-ZatsepinKuz'min volume is high enough, so no markedly anisotropic distribution of UHECRs is expected which is perfectly in line with the current observations. Thus, the giant flares of the AGNs similar to ones observed with the Fermi LAT experiment can be primary sources of the UHECRs with energies $E>10^{20} \mathrm{eV}$.

\section{Acknowledgements.}

This work was supported by RSF research project No. 14-12-00146. The authors want to thank Andrei Gruzinov, Oleg Kalashev and Sergey Troitsky for fruitful discussions. The analysis is based on data and software provided by the Fermi Science Support Center (FSSC). This research has made use of NASA's Astrophysics Data System.

\section{References}

[1] K. Greisen, Physical Review Letters 16, 748 (1966)

[2] G.T. Zatsepin, V.A. Kuz'min, Soviet Journal of Experimental and Theoretical Physics Letters 4, 78 (1966)

[3] R.U. Abbasi et al. (HiRes), Phys. Rev. Lett. 100, 101101 (2008), astro-ph/0703099

[4] J. Abraham et al. (Pierre Auger), Phys. Rev. Lett. 101, 061101 (2008), 0806.4302

[5] T. Abu-Zayyad et al. (Telescope Array), Astrophys. J. 768, L1 (2013), 1205. 5067

\footnotetext{
${ }^{4}$ Present method of composition studies use data obtained by detectors of fluorescent light from extended showers [35] and these detectors have livetime of only $10 \%$
} 
[6] A.M. Hillas, ARA\&A22, 425 (1984)

[7] E. Waxman, Physical Review Letters 75, 386 (1995), astro-ph/9505082

[8] E. Waxman, A. Loeb, JCAP8, 026 (2009), 0809. 3788

[9] G.R. Farrar, A. Gruzinov, ApJ693, 329 (2009), 0802 . 1074

[10] C.D. Dermer, Are Gamma-Ray Bursts the Sources of the Ultra-High Energy Cosmic Rays?, in American Institute of Physics Conference Series, edited by J.E. McEnery, J.L. Racusin, N. Gehrels (2011), Vol. 1358 of American Institute of Physics Conference Series, pp. 355-360, 1103.4834

[11] R.S. Nemmen, M. Georganopoulos, S. Guiriec, E.T. Meyer, N. Gehrels, R.M. Sambruna, Science 338, 1445 (2012), 1212.3343

[12] S. Abdollahi, M. Ackermann, M. Ajello, A. Albert et al., ArXiv e-prints (2016), 1612.03165

[13] G. Ghisellini, F. Tavecchio, MNRAS448, 1060 (2015), 1501.03504

[14] T. Savolainen, D.C. Homan, T. Hovatta, M. Kadler, Y.Y. Kovalev, M.L. Lister, E. Ros, J.A. Zensus, A\&A512, A24 (2010), 0911. 4924

[15] O. Hervet, C. Boisson, H. Sol, A\&A592, A22 (2016), 1605.02272

[16] R. Aloisio, P. Blasi, I. De Mitri, S. Petrera, ArXiv e-prints (2017), 1707.06147

[17] F.W. Stecker, Physical Review Letters 21, 1016 (1968)

[18] C. Patrignani et al. (Particle Data Group), Chin. Phys. C 40, 100001 (2016)

[19] The Pierre Auger Collaboration, A. Aab, P. Abreu, M. Aglietta, E.J. Ahn, I.A. Samarai, I.F.M. Albuquerque, I. Allekotte, P. Allison, A. Almela et al., ArXiv e-prints (2015), 1509.03732

[20] E.G. Berezhko, D.C. Ellison, ApJ526, 385 (1999)

[21] A. Meli, P.L. Biermann, A\&A556, A88 (2013), 1207.4397

[22] N. Globus, D. Allard, R. Mochkovitch, E. Parizot, MNRAS451, 751 (2015), 1409. 1271

[23] A.M. Bykov, D.C. Ellison, S.M. Osipov, Phys. Rev. E95, 033207 (2017), 1703.01160

[24] K. Ptitsyna, A. Neronov, A\&A593, A8 (2016), 1510.04023

[25] O. Kalashev, Constraining Dark Matter and Ultra-High Energy Cosmic Ray Sources with Fermi-LAT Diffuse Gamma Ray Background, in European Physical Journal Web of Conferences (2016), Vol. 125 of European Physical Journal Web of Conferences, p. $02012,1608.07530$

[26] S.V. Troitsky, Soviet Journal of Experimental and Theoretical Physics Letters 96, 13 (2012), 1205.6435

[27] M.S. Pshirkov, P.G. Tinyakov, F.R. Urban, MNRAS436, 2326 (2013), 1304 . 3217

[28] M.S. Pshirkov, P.G. Tinyakov, P.P. Kronberg, K.J. Newton-McGee, ApJ738, 192 (2011), 1103.0814

[29] K. Murase, H. Takami, ApJ690, L14 (2009), 0810. 1813

[30] H. Yüksel, T. Stanev, M.D. Kistler, P.P. Kronberg, ApJ758, 16 (2012), 1203.3197

[31] M.S. Pshirkov, P.G. Tinyakov, F.R. Urban, Physical Review Letters 116, 191302 (2016), 1504.06546

[32] R.U. Abbasi et al. (Telescope Array), Astropart. Phys. 64, 49 (2015), 1408. 1726

[33] Pierre Auger Collaboration, ArXiv e-prints (2014), 1409. 5083

[34] The Telescope Array Collaboration, The Pierre Auger Collaboration, ArXiv e-prints (2018), 1801.01018

[35] T. Abu-Zayyad et al. (Telescope Array), Astropart. Phys. 48, 16 (2013), 1305.6079 